\title{
CONTRAST SENSITIVITY AT HIGH VELOCITIES
}

\author{
DAvid C. BuRR* and JoHn Rosst \\ Istituto di Neurofisiologia del CNR, Via S. Zeno 51, 56100 Pisa. Italy
}

(Received in revised form 9 June 1981)

\begin{abstract}
Abstmet-Measurements were made of the contrast required to see the direction of motion of drifting gratings (Part 1) and of moving bars (Part 2). The spatial frequency at which least contrast is required to see sinusoidal gratings decreases as their velocity increases, but peak sensitivity is identical at all velocities up to $800 \mathrm{deg} / \mathrm{sec}$. Similarly, the wider a single bar, the higher the velocity at which it is best visible. A bar $80 \mathrm{deg}$ wide is best seen when moving at $300-500 \mathrm{deg} / \mathrm{sec}$, and can be seen, and its direction of motion identified, even when moving at $10^{4} \mathrm{deg} / \mathrm{sec}$. These results show that motion does not diminish the visual passband, but instead slides the spatial frequency window along the spatial frequency scale. maintaining peak sensitivity at a temporal frequency of about $10 \mathrm{~Hz}$ (at photopic luminances).
\end{abstract}

\section{INTRODUCTION}

When the eyes are stationary, and light ample, the human visual system peaks in sensitivity at a spatial frequency of about $3 \mathrm{c} / \mathrm{deg}$ (Campbell and Robson, 1968). Image motion and temporal flicker both alter visual sensitivity to contrast. Studies with gratings flickered in counterphase (e.g. Robson, 1966; Kelly. 1971) show that the form of the contrast sensitivity function is markedly changed by the temporal frequency at which spatial frequency components are flickered. In particular, gratings of low spatial frequency become more visible as their temporal frequency is increased, up to about $10 \mathrm{~Hz}$. Here we examine the implications of the change of the form of the contrast sensitivity function for the visibility of drifting gratings of low spatial frequency and of moving bars of large size.

It is a commonly held assumption that the human visual system cannot resolve objects moving at high speeds (Dodge, 1900: Woodworth, 1906: Richards, 1969; Matin, 1974; Kelly, 1979a, b). As a typical example, Matin (1974) reports that although it has been shown that cat visual neurones respond to velocities of $100 \mathrm{deg} / \mathrm{sec}$ (e.g. Cleland et al., 1971) "at least in the human visual system such velocities do not elicit a sensation that an object is moving" (p. 909). However, considering how widely this belief is held, and the fact that neurones in both the cat and monkey are capable of responding to very high velocities (e.g. Cleland et al., 1971: Wurtz, 1969), there is surprisingly little psychophysical evidence to support it. Many early investigators observed that at speeds above $100 \mathrm{deg} / \mathrm{sec}$. Landolt Cs or Snellen letters are not seen (e.g. di Silva. 1929). More recently Brown (1958) reported that a small spot of light is not visible

\footnotetext{
* Present address: Department of Psychology, University of Western Australia, Nedlands WA 6009 . Australia.

+ On leave from the Department of Psychology, University of Western Australia.
}

when it is moving faster than $32 \mathrm{deg} / \mathrm{sec}$. On the other hand, Pollock (1953) and Johnstone and Riggs (1979), using larger test stimuli, have measured velocity thresholds more than an order of magnitude higher.

We measure contrast sensitivity to stimuli in rapid motion, firstly with periodic sinusoidal gratings caused to drift and secondly, with single biphasic bars consisting of one cycle of sinusoidal grating, caused to move. In all studies the observer is required to detect the direction of motion, not merely the presence of the stimulus on the screen. Thus it is motion rather than flicker thresholds which are measured.

\section{PART 1: DRIFTING GRATINGS}

In the first series of studies, we measured contrast sensitivity to sinusoidal gratings caused to drift at image speeds ranging from 0 to $800 \mathrm{deg} / \mathrm{sec}$. To bring out more clearly the effect of image motion, each set of measurements was made at constant speed, rather than at constant temporal frequency which is more conventional (Robson. 1966; Van Ness et al., 1967; Kelly, 1971). Thus, as we varied the spatial frequency $F_{\text {s }}$ of the gratings, we varied also the temporal frequency $F_{\text {, }}$ so that the ratio $F_{t} / F_{\text {s }}$ (i.e. the speed) remained constant.

\section{Methods}

Measurements were made both in Cambridge and Pisa. Computer generated gratings were suitably filtered and displayed on the face of a cathode ray oscilloscope at $150 \mathrm{frames} / \mathrm{sec}, 1000$ lines/frame, using the standard television raster technique of Schade (1956). The visible screen was a circle of $20 \mathrm{~cm}$ diameter, centered within a $1 \mathrm{~m}^{2}$ surround, floodlit to the same mean luminance as the screen $\left(200 \mathrm{~cd} / \mathrm{m}^{2}\right)$. In order to encompass a wide range of spatial frequencies, viewing distance and hence field size of the screen was varied from session to session. Measurements within the velocity range of $0-1 \mathrm{deg} / \mathrm{sec}$ were 


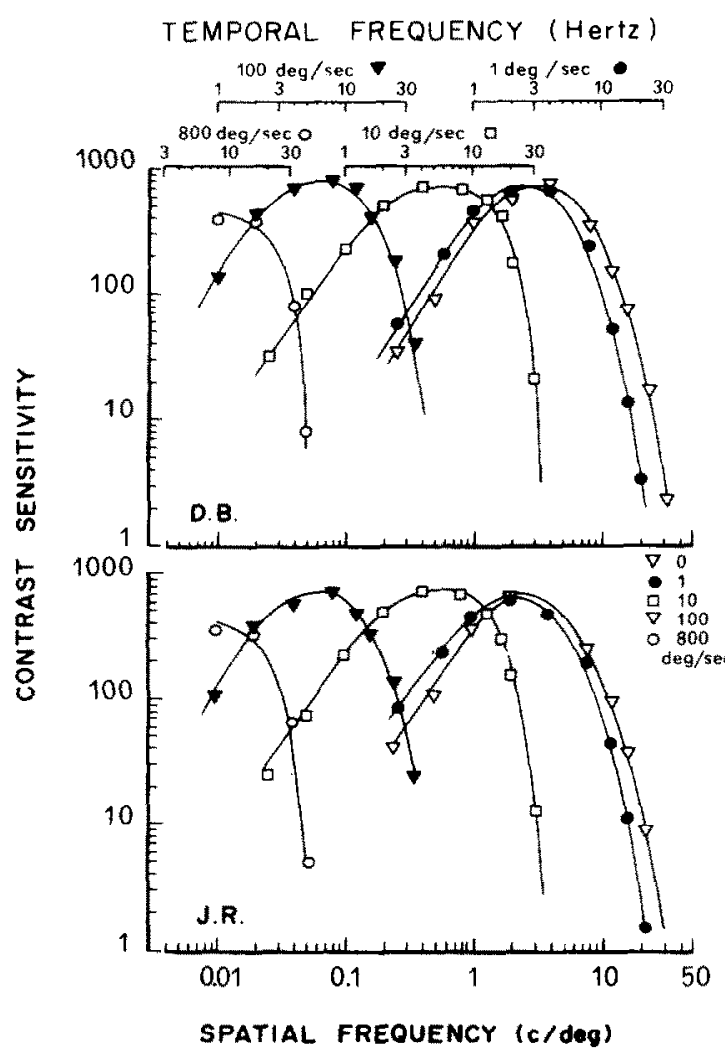

Fig. 1. Contrast sensitivity curves for the two observers for sinusoidal gratings drifting at various speeds. The lower abscissae shows the spatial frequency of the gratings, and the upper abscissae the corresponding temporal frequency for each drift speed. Note that at all drift speeds, the curves have the same height, width and general shape.

made at $70 \mathrm{~cm}$ (screen size $16 \mathrm{deg}$ ) and the remainder at $7 \mathrm{~cm}$ (screen size $110 \mathrm{deg})^{*}$.

Waveforms, and their positioning frame by frame, were provided by a small laboratory computer (PDP-8/1 for the Cambridge measurements and PDP-11/03 for the Pisa measurements), which also randomized trial conditions (including direction of drift) and recorded and averaged threshold measurements. Thresholds were determined by the method of adjustment, each data point being the mean of five separate settings. To minimize sterotyping of responses, the contrast was set before each trial to a random level unknown to the observer by a computer controlled logarithmic attenuator.

Gratings were vertical and drifted right or left, direction being randomized on each trial. Eye movements were minimized by instructing the observers to fixate a large $(1 \mathrm{~cm})$ black central fixation mark. Thresholds were set to the minimum contrast at which the direction of drift could be seen. Thus the curves reflect thresholds for motion detection, not

* Such close viewing distance will of course introduce considerable geometic distortions of the display but this should not significantly affect the form of the results (see also Fig. 5). flicker-fusion. However, it should be noted that the thresholds for flicker do not differ greatly from those for motion in these conditions, as one would expect from the similarity in thresholds of drifting and counterphased gratings (Levinson and Sekular, 1975; Watson et al., 1980).

Results are reported for two observers, the authors. Viewing was monocular, with the left eye patched.

\section{Results and discussion}

Threshold measurements for five different drift speeds are summarized in Fig. 1. The curves all have the same general form. The height is in each instance about the same, indicating the same peak sensitivity at all velocities, and all curves have the same general width, with similar low frequency and high frequency attenuation. What changes with drift speed is the position of the curves on the spatial frequency axis: the higher the velocity, the lower the spatial frequency at which the curves peak. At a velocity of $100 \mathrm{deg} / \mathrm{sec}$, the curves peak at about $0.06 \mathrm{c} / \mathrm{deg}$; and at this frequency, sensitivity is as high as it is for a stationary grating of $3 \mathrm{c} / \mathrm{deg}$. Thus, a grating which is visible only at high contrast when the image is still becomes visible at minimal contrast when the image moves at the correct velocity.

The upper abscissa of Fig. 1 shows the temporal frequency (i.e. the rate at which the grating bars pass a given point) associated with each spatial frequency and velocity $\left(F_{t}=F_{s} \cdot v\right)$. From these axes it can be seen that the temporal envelope varies little with drift speed. Figure 2 dipicts this consistency more clearly, by plotting the same data on axes of temporal rather than spatial frequency. Whereas the peaks of the spatial curves of Fig. 1 spread over a range of about three logarithmic units, the peaks of the temporal curves of Fig. 2 are nearly coincident, varying by only about a half of a logarithmic unit. It is only at the lowest velocity, $1 \mathrm{deg} / \mathrm{sec}$, that there is a small peak shift, and here the results may have been influenced by small eye movements (cf. Kelly, 1979a, b).

At every velocity measured in this experiment, the upper limit for motion perception is set by the temporal frequency of image modulation. Velocity itself is

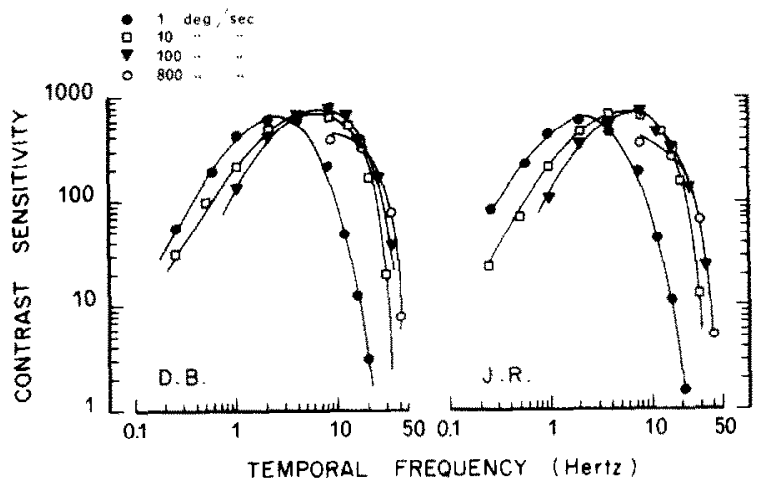

Fig. 2. The same measurements as Fig. 1 plotted against temporal rather than spatial frequency. 
irrelevant. The form of the curves of Fig. 2 suggests that signals from moving patterns are all passed through temporal filters of roughly the same shape, and that motion can be perceived provided that the limits of the temporal filters are not exceeded. But, as Fig. 1 shows, the assosciated spatial filters must encompass an enormous range, extending far further down the spatial frequency spectrum than most investigators care to go; and when stimuli of these very low spatial frequencies are moving so as to modulate at their optimal temporal frequency, they are moving very fast indeed.

The consistency of the form of the curves of Fig. 2 is interestingly reflected in the fact that the very fast velocities never appear particularly fast. At $800 \mathrm{deg} /$ sec (and in fact even at velocities as high as $3000 \mathrm{deg} /$ sec), a low frequency grating of modest temporal frequency appears to glide past the field of view smoothly and unhurriedly. And, at all temporal frequencies, the apparent speed remains unchanged with viewing distance, which alters both spatial frequency and velocity but leaves temporal frequency unchanged. These observations suggest that perceived velocity is computed from the temporal frequency of signal modulation, not retinal velocity, thereby maintaining velocity constancy over a wide range of viewing conditions. This point requires further investigation.

\section{PART 2: MOVING BARS}

The results of Part 1 show that image motion, no matter how swift (within rather broad experimental limits), does not impair sensitivity to gratings but merely changes the frequency spectrum to which the visual system responds, in effect sliding the window of visibility to a lower position on the spatial frequency axis, without changing its form. Motion has two effects: it deemphasizes or "smears" high frequency components, but also emphasizes low spatial frequency image components, that is components of large size, which are virtually invisible when the image is still.

It might be thought that the low frequency results do not extend to single objects, as gratings have an unlimited number of cycles moving past the retina at a steady flicker rate. And, as previously mentioned, it is widely beleived that object motion is invisible at image velocities above $100 \mathrm{deg} / \mathrm{sec}$. Therefore, we considered the visibility of objects: single cycles of bars of grating as they move in isolation across a screen of the same mean luminance as the bar itself.

\section{Methods}

Single vertical bars, comprising one cycle of sine wave grating starting at zero phase, were displayed on the face of a cathode ray oscilloscope at 1000 frames/ sec with free running $3 \mathrm{MHz}$ vertical raster. The visible screen was a $5 \times 5 \mathrm{~cm}$ square of mean luminance $20 \mathrm{~cd} / \mathrm{m}^{2}$, with a central black fixation mark of

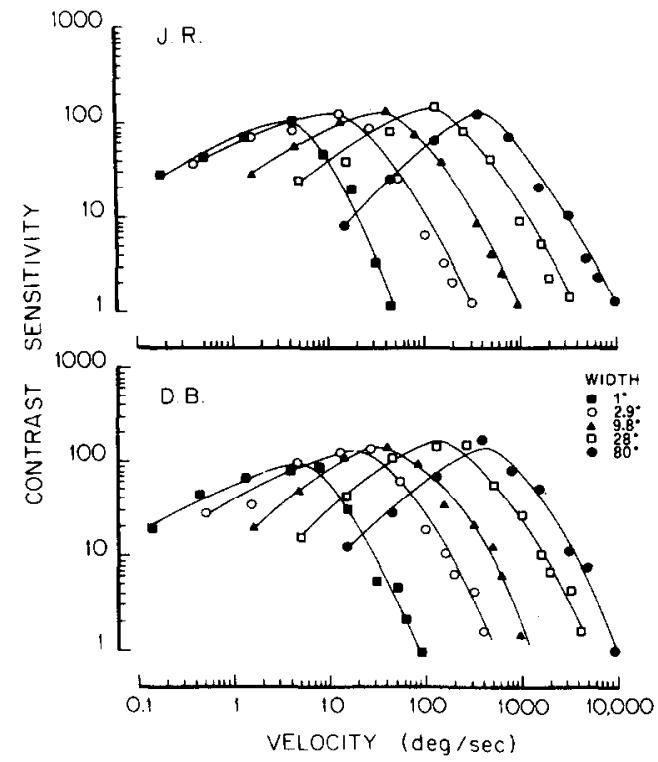

Fig. 3. Contrast sensitivity to single cycle, biphasic sinusoidal bars of various width as a function of image velocity.

$5 \mathrm{~mm}$ diameter. To encompass a wide range of bar widths, viewing distance (and hence field size of the screen) was varied from session to session, over a range from $3 \mathrm{~cm}$ to $3 \mathrm{~m}$. At the nearer viewing distances, the image was brought into focus by a positive lens. All viewing was monocular with left eye patched. Each bar was formed by a function generator (Interstate Electronic Corporation F34), triggered by computer (PDP 11-03) at a suitable delay after the start of each frame. The delay could be incremented or decremented by the computer (equipped with a $1 \mathrm{MHz}$ digital clock) so as to cause the bar to be slightly displaced on each frame, and hence to appear to move smoothly either rightwards or leftwards across the screen at any desired velocity. Step size was sufficiently small in relation to bar width for motion to remain smooth in appearance even at the highest velocities. It also meant that the time averaged luminance across the screen was almost constant, the biphasic bar leaving no visible aftertrace. After the passage of each bar, their was a $0.5 \mathrm{sec}$ pause before a new bar appeared.

As before, thresholds were determined by method of adjustment, but key data points were checked with forced choice methods. Threshold was defined as the minimum contrast at which the direction of the motion of the bar could be seen. Velocity was calculated at the point of fixation, and applies strictly only at this point. since the distortions resulting from a flat screen are not negligible at the closest viewing distance.

\section{Results and discussion}

Threshold measurements for five bar widths are summarized in Fig. 3, as a function of image speed. 


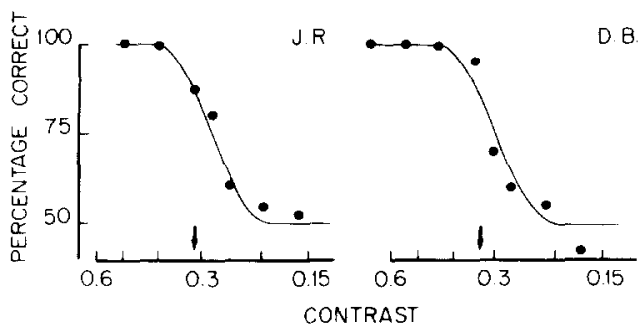

Fig. 4. Forced choice measurements to confirm the visibility of bars moving at $10,000 \mathrm{deg} / \mathrm{sec}$. The two observers were required to report the direction of motion (rightward or leftward drift) of a bar of width $\mathbf{8 0}$ caused to move at $10,000 \mathrm{deg} / \mathrm{sec}$ (viewing distance $3 \mathrm{cms}$ ). Each point is the average of $\mathbf{4 0}$ trials, collected over four brief sessions. The computer randomized the conditions (including direction of drift), presented the trials and scored and averaged the response. The arrows point to thresholds for the two ob-

servers, corresponding to the $83 \%$ correct response

All curves have comparable peak values, indicating comparable sensitivity at best velocity for all bar widths. However, the shape of the curves changes systematically with bar width, having a somewhat shallower low velocity cut at smaller bar widths.

The most significant feature of the data is that, as bar width increases, the velocity at which sensitivity peaks also increases. For the widest bar $(80 \mathrm{deg})$ the velocity peak is to be found in the range of $300-500 \mathrm{deg} / \mathrm{sec}$, well above the value of $100 \mathrm{deg} / \mathrm{sec}$, which is often taken to be the limiting value for the perception of objects in motion. However, bars which are $1 \mathrm{deg}$ wide are invisible at any contrast when moving at speeds in excess of $100 \mathrm{deg} / \mathrm{sec}$.

Forced choice measurements. The widest bar is easily visible, at high contrast, when moving at $10,000 \mathrm{deg} / \mathrm{sec}$. To confirm that objects are in fact visible at these velocities, this last point of the curve for the $80 \mathrm{deg}$ bar was checked by a two alternative forced choice method, in which the observer was required to report the direction of motion of the bar, which moved, on each trial, either left or right at random. The results, summarized in Fig. 4, show that the method of adjustment measurements are if anything conservative, as the forced choice thresholds for seeing the direction of motion (2.8 and 3.5 for D.B. and J.R. respectively at $83 \%$ correct) are slightly higher than those of Fig. 4 (both being 1).

A reviewer has raised the possibility that observers may have been tracking the moving stimuli, thereby reducing the retinal image velocity, which would of course trivilize our results. While eye tracking may be possible (but not probable, given the heavy fixation mark) for the measurements of Fig. 1 where the stimulus was continuously visible, it is not possible under these conditions of brief stimulus presentation. For each trial the whole display was completed by $16 \mathrm{msec}$, well within the latency for pursuit eye movements (Westheimer, 1954).
Effect of viewing distance. The scrupulous reader might raise several objections at this point. Firstly, because of the flat screen, there is considerable variation in angular velocity across the screen at the closer viewing distances. Secondly, the distance traversed by the bar is short in comparison to the width of the bar (about one bar width) and the time for which the bar is visible varies inversely with velocity at each viewing distance. To investigate these possible sources of perturbation, we remeasured visibility thresholds for the $28 \mathrm{deg}$ bar at two viewing distances, 3 and $10 \mathrm{~cm}$, using the same $5 \mathrm{~cm}$ square mask for the oscilloscope face. Thus at $10 \mathrm{~cm}$, the bar size was $5 \mathrm{~cm}, 28 \mathrm{deg}$, and the field size $28 \mathrm{deg}$, while at $3 \mathrm{~cm}$ the bar size was $1.5 \mathrm{~cm}, 28 \mathrm{deg}$, and the field size $80 \mathrm{deg}$. At this closer viewing distance, the bar moved for about three times as long as for the further distance.

Figure 5 summarizes the results. The close agreement between the two curves obtained at different distances suggests that neither size and velocity distortions resulting from the flat screen, nor variability in the duration of the motion trajectory meterially distort the form of the data. The slight differences between the curves can probably be put down to probability summation which works in favour of the bar that moves over the longer path (Sachs et al. 1971; Watson, 1979).

The results of this section are quite consistent with those of Part 1. For example. consider the results of the $80 \mathrm{deg}$ bar (Fig. 3). An $80 \mathrm{deg}$ bar has a fundamen-

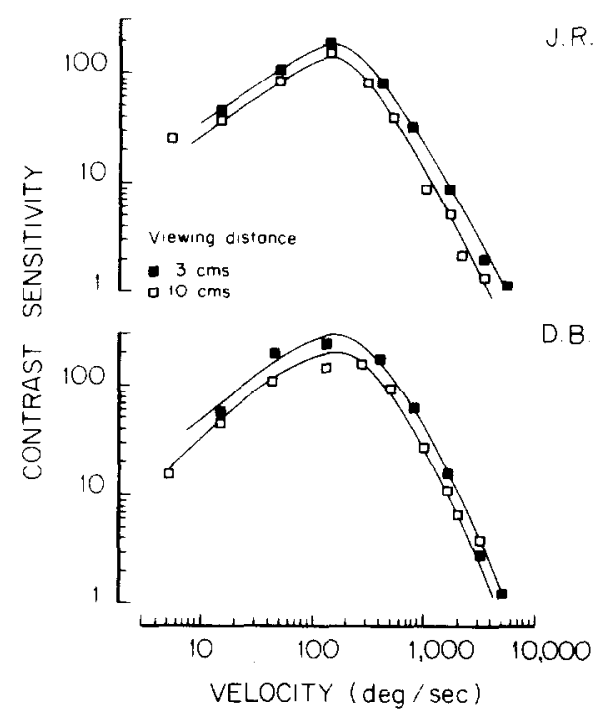

Fig. 5. Contrast thresholds for a $28 \mathrm{deg}$ moving bar at two viewing distances, 3 and $10 \mathrm{~cm}$, and hence two different field widths, 80 and $28 \mathrm{deg}$ respectively. The bar traversed a distance equal to about three bar widths at the close viewing distance, but only about one bar width at the farther viewing distance. The close agreement of the two curves suggests that neither geometical distortions resulting from a flat screen and close viewing distance, nor extent of excursion of the target alter significantly the form of the results. 
tal frequency of $0.0125 \mathrm{c} / \mathrm{deg}$. Thus the peak velocity for this bar, $300-500 \mathrm{deg} / \mathrm{sec}$, corresponds to a temporal frequency of about $6 \mathrm{~Hz}$ for the fundamental, in agreement with the curves of Fig. 2 (small discrepancies between these results can probably be accounted for by the difference in mean lumininace for the two sets of measurements). Spatial frequency components are visible up to temporal frequencies of $50 \mathrm{~Hz}$. At an image speed of $10,000 \mathrm{deg} / \mathrm{sec}$ (when the $80 \mathrm{deg}$ bar is just visible) only those components of $0.005 \mathrm{c} / \mathrm{deg}$ and below will be visible. Huwever, a single bar has a wide spread of spatial components including some at much lower frequencies than the fundamental*. Thus the $80 \mathrm{deg}$ bar comprises not only its fundamental of $0.0125 \mathrm{c} / \mathrm{deg}$, but also components of much lower spatial frequency (extending below $0.005 \mathrm{c} / \mathrm{deg}$ ) which, even at image speeds of $10,000 \mathrm{deg} / \mathrm{sec}$, modulate at temporal frequencies below $50 \mathrm{~Hz}$. These low spatial frequency components presumably render the bar visible at high velocities.

\section{GENERAL DISCUSSION}

It is well established, and now widely understood, that stationary scenes, observed in good light with the resting eye, are seen through a "spatial frequency window". It has been assumed that motion lowers and narrows the visual passband. For example, Kelly (1979) says: "at velocities greater than $100 \mathrm{deg} / \mathrm{sec}$ there would be no contrast sensitivity at any spatial frequency within the range of normal vision" (p. 1344). We have shown that image motion does not alter the band pass characteristics of vision, neither by lowering maximum sensitivity, nor by narrowing the band itself. Rather, the effect of image motion is to slide the spatial frequency window down the spatial frequency scale, diminishing neither its height nor its width. Sensitivity is maintained, and so too is the range of visible spatial frequencies.

Part 2 shows that the visibility objects in motion may be understood in terms of the visibility of their spatial frequency components. The direction of motion of large objects, which contain low spatial frequency components, can be resolved at high velocities at which smaller objects are invisible. For example, a bar $1 \mathrm{deg}$ in width becomes invisible at $100 \mathrm{deg} / \mathrm{sec}$ however high its contrast, but wider bars are easily seen at this velocity.

All thresholds reported here are motion thresholds, the minimum contrast required to see the direction of motion. At threshold, one seems to have a pure sensation of motion, that is objectless motion (Wertheimer,

\footnotetext{
* The spectrum of spatial frequencies $(F(w))$ of a single cycle of sine wave is given by:

$$
F(w)=\frac{A w_{0}}{\pi} \frac{1}{w_{0}^{2}-w^{2}} \sin \frac{2 \pi w}{w_{0}}
$$

where $A$ is the amplitude, and $w$ the fundamental spatial frequency of the single cycle.
}

1912) with no clear impression of what it is that is moving (Keesey, 1972; Kulikowski and Tolhurst, 1973). However, at all spatial frequencies and at all bar widths employed in these experiments, it was necessary to raise the contrast by only a few decibels above the threshold for pure motion in order to see clearly the spatial structure of the grating or the form of the sinusoidal bar. This casts some doubt upon the popular notion that there are two independent visual systems spanning different spatial frequency ranges, one analyzing pattern and the other motion (e.g. Tolhurst, 1973; Kulikowski and Tolhurst, 1973: Kulikowski, 1978). Gratings of spatial frequency $0.01 \mathrm{c} / \mathrm{deg}$, an order of magnitude below the supposed lower limit of the pattern system (e.g. Tolhurst, 1973), have a clear spatial structure at appropriate drift speeds, even at low contrast. It seems more probable that the detectors which respond to these low frequencies are organized so as to signal both pattern and motion (Burr, 1979, 1980, 1981; Lennie, 1980; Derrington and Henning, 1981).

Image velocites in excess of $100 \mathrm{deg} / \mathrm{sec}$ are the usual result of saccadic eye movements, which occur frequently in natural viewing. Much speculation on the problem of saccadic suppresion, or saccadic ommission (failure to see a visual grey out) has supposed that saccadic speeds are too fast for the visual system to resolve and therefore result in a complete blurring or "grey out" of the visual scene (e.g. Dodge, 1905; Woodworth, 1938; MacKay, 1973; Campbell and Wurzt, 1978). However, most previous measurements of vision during saccades have employed targets of high spatial frequency or small size, which, as our results show, will be rendered invisible at saccadic velocities. On the other hand, low spatial frequency components, not easily visible when the image is stationary, are made more visible and can readily be resolved when the image moves at saccadic speeds. Why then is the observer not startled during a saccade by the sudden intrusion of low frequency components onto the scene? A subsequent study suggests that during saccades motion sensitivity is dampened, precisely to avoid the disturbing consequences of saccadic image motion which would follow if it were left intact (Burr et al., 1981).

Acknowledgements - We thank Dr Fergus Campbell, of Cambridge and Professor Lamberto Maffei, of Pisa, for the use of their laboratory facilities. D.B. was supported by a Hackett Fellowship from the University of Western Australia when at Cambridge and a Royal Society European Exchange Fellowship when at Pisa.

\section{REFERENCES}

Brown R. H. (1958) Influence of stimulus luminance upon the upper speed threshold for the discrimination of movement. J. opt. Soc. Am. 48, 125-128.

Burr D. C. (1979) On the perception of objects in motion. Ph.D. thesis Univ. Cambridge U.K.

Burr D. C. (1980) Motion smear. Nature 284, 164-165.

Burr D. C. (1981) Visual summation of objects in motion. Proc. $R$. Soc. B 211, 321-339. 
Burr D. C., Johnstone J. R., Holt J. and Ross J. (1981) Selective depression of motion selectivity during saccades. To be published.

Campbell F. W. and Robson J. G. (1968) Applications of Fourier analysis to the visibility of gratings. J. Physiol. 197, 551-566.

Campbell F. W. and Wurtz R. H. (1979) Saccadic omission: why we do not see a grey out during a saccadic eye movement. Vision Res. 18, 1297-1303.

Cleland B., Levick W. and Sanderson E. W. (1973) Properties of sustained and transient cells in the cat retina. $J$. Physiol. 228, 649-680.

Derrington A. M. and Henning G. B. (1981) Pattern discrimination with flickering stimuli Vision Res. 21, $597-602$.

Dodge R. (1905) The illusion of clear vision during eye movements. Psychol. Bull. 2, 193-199.

Johnstone J. R. and Riggs L. A. (1979) Upper velocity threshold for the detection of movement. Optics Lett. 4, 309-310.

Keesey U. T. (1972) Flicker and pattern detection: a comparison of thresholds. J. opt. Soc. Am. 62, 446-448.

Kelly D. H. (1971) Theory of flicker and transient responses. J. opt. Soc. Am. 61, 537-546; 632-640.

Kelly D. H. (1979a) Motion and vision. I. Stabilized images of stationary gratings. J. opt. Soc. Am. 69, 1266-1339.

Kelly D. H. (1979b) Motion and vision. II. Stabilized spatio-temporal threshold surface. J. opt. Soc. Am. 69, 1340-1349.

Kulikowski J. J. (1978) Pattern and movement detection in man and rabbit. Vision Res. 18, 183-189.

Kulikowski J. J. and Tolhurst D. J. (1973) Psychophysical evidence for sustained and transient neurons in the human vision system. $J$. Physiol. 232, 149-162.

Lennie P. (1981) Perceptual signs of parallel pathways Phil. Trans. R. Soc. B 290, 23-37.

Levison E. and Sekuler R. (1975) The independence of channels in human vision selective for the direction of movement. J. Physiol. 250, 347-366.

MacKay D. (1973) Visual stability and voluntary eye movements. Handbook of Sensory Physiology, Vol. VII/3. Springer-Verlag, Berlin.

Matin E. (1974) Saccadic supression: a review and an analysis. Psychol. Bull. 81, 899-917.

Pollock W. T. (1953) The visibility of a target as a function of its speed of movement. J. exp. Psychol. 45, 449-454.

Richards W. (1969) Saccadic suppression. J. opt. Soc. Am. $59,617-623$

Robson J. G. (1966) Spatial and temporal contrast sensitivity functions of the visual system. J. opt. Soc. Am. 56, 1141-1142.

Sachs M. B., Nachmias J. and Robson J. G. (1971) Spatial frequency channels in human vision. .I. opt. Soc. Am. 61, 1176-1182.

Schade O. H. (1956) Optical and photoelectric analogue of the eye. J. opt. Soc. Am. 46, 721-739.

Tolhurst D. J. (1973) Separate channels for the analysis of the shape and the movement of a moving visual stimulus. J. Physiol. 231, 385-402.

Van Ness F. L., Koenderink J. J., Nas H. and Bouman M. A. (1967) Spatiotemporal modulation transfer in the eye. J. opt. Soc. Am. 57, 1082-1088.

Watson A. B. (1979) Probability summation over time. Vision Res. 19, 515-522.

Watson A. B., Thompson P. G., Murphy B. J. and Nachmias J. (1980) Summation and discrimination of gratings moving in opposite directions Vision Res. 20, 341-348.

Westheimer G. (1954) Mechanism of saccadic eye movement. A.M.A. Archs. Opthal. 52, 710-724.

Woodworth R. S. (1938) Experiment Psychology. Holt, New York.

Wurtz R. H. (1976) Visual receptive fields of striate cortex neurons in awake monkeys. $J$. Neurophysiol. 32, $727-742$. 